\title{
Moderne Biotechnologie und Demokratie
}

\section{St. Albrecht: Freiheit, Kontrolle und Verantwortlichkeit in der Gesellschaft. Moderne Biotechnologie als Lehrstück. Hamburg: Hamburg University Press, 461 S., ISBN 3-937816-16-X, $35 \epsilon^{1}$}

\section{von Rolf Meyer, ITAS}

Dieses Buch über Biotechnologie und Demokratie beruht auf der Habilitationsschrift von Stephan Albrecht. Das vorliegende Werk basiert dabei auf seiner langjährigen Forschungsarbeit zu den Entwicklungen in der modernen Biotechnologie. Die Analyse stellt die Frage der Verantwortung in den Mittelpunkt und steht damit im Gegensatz zu den vielen vorliegenden Arbeiten zu den Chancen und Risiken der modernen Biotechnologie. Fragen der Interessen, Macht und politischen Organisation werden behandelt, die in wissenschaftlichen Analysen und Politik beratenden Studien zu ökonomischen Chancen und ökologischen und gesundheitlichen Risiken oft zu kurz kommen.

Ein Ergebnis der umfangreichen Analyse ist, dass Verantwortung meistens „die anderen“ tragen. Politische, rechtliche, administrative und innerwissenschaftliche Kommunikations- und Handlungsstrukturen verhindern oder verdünnen Verantwortung bis zur Unauffindbarkeit. Es wird nachgewiesen, dass der biotechnologische Fortschritt nicht wissenschaftsimmanent erfolgt, sondern vornehmlich politisch gestaltet ist. Einen grundlegenden Fehler in diesem Fortschritt sieht Stephan Albrecht darin, dass dieser nicht angemessen öffentlich, also als Res publica verstanden und verhandelt wird. In dieser Arbeit bleibt es aber nicht nur bei einer Beschreibung und Analyse der Defizite, sondern der Autor macht auch Vorschläge für eine institutionelle Ausprägung der Wahrnehmung von Verantwortung in den und für die Wissenschaften.

Im ersten Teil des Buches wird zunächst der Untersuchungsgegenstand der modernen Biotechnologie (und seine Anwendungsfelder in Medizin, Landwirtschaft und Umwelttechnik) umrissen. Dann werden charakteristische Modi des Umgangs mit der modernen Biotechnologie untersucht. Behandelt werden dabei vier Bereiche: wissenschaftliche Dispute, Regulierungen, Technikfolgen-Abschätzung (bzw. Versuche einer wissenschaftlich unterstützten Reflexion als Mittel der gesellschaftspolitischen Steuerung) und schließlich Gestaltungsarrangements und ,policy coalitions“. Der Vergleich dieser Modi in Deutschland und den USA, vor allem aber das Nachzeichnen der historischen Entwicklung seit den 70er Jahren des letzten Jahrhunderts - was selbst einem Leser mit wissenschaftlichem Schwerpunkt und viel Erfahrung im Bereich der Gentechnik in der Zusammenschau und in vielen Details nicht so präsent bzw. bekannt ist - machen diese Kapitel äußerst interessant.

Aufbauend auf diesem Überblick wird im zweiten Teil der Arbeit die kontroverse Geschichte der modernen Biotechnologie, untergliedert in mehrere Themenkreise, in vielen Facetten beleuchtet. Die Hauptthemenkreise sind die Entstehung politischer Normierungen und technologiepolitischer Steuerungen, Science und Technology Assessment sowie demokratiepolitische und -theoretische Implikationen, jeweils mit vielen Verästelungen. Dabei handelt sich um schon als Einzelaufsätze publizierte Arbeiten, die über einen längeren Zeitraum entstanden sind und nur begrenzt überarbeitet wurden. Die eine oder andere Redundanz ist dadurch zwangsläufig gegeben. Somit ist dieser Teil nicht unbedingt zum systematischen Durchlesen geeignet, aber auf jeden Fall ein Schatzkästchen an Informationen, Einordnungen und Einschätzungen.

Im dritten und letzten Teil werden die verschiedenen Stränge der Argumentation wieder zusammengeführt. Dabei geht es um die Spannungen zwischen Wissenschaft, biotechnologischer Politik und Gesellschaft, um Verantwortung als grundlegendes Element zur Bewahrung der Freiheit in den Wissenschaften und um die Erneuerung von Demokratie. Unter ,Verantwortungsorganisation" werden auch eine Vielzahl von politisch-praktischen Vorschlägen unterbreitet und begründet, wie in der Wissenschaft, in forschenden Unternehmen und den dazugehörigen Bereichen der öffentlichen Politik Identifikations-, Priorisierungs-, Herstellungs-, Implikations- und Evaluationsverantwortung miteinander wahrgenommen werden können.

Aus dem Resümee sei hervorgehoben, „dass die Fragen nach einer Veränderung des 
Weges im Umgang mit der modernen Biotechnologie, mit Freiheit, Kontrolle und Verantwortlichkeit, nicht solche nach einer veränderten Wissenschaft und Wissenschaftspolitik, sondern solche nach einer veränderten Gesellschaftspolitik, also nach Demokratie, sind.“ (S. 405). Albrecht bemerkt weiter: „Für die zukünftigen Demokratiefragen, die von der modernen Biotechnologie ausgehen, ist die sich erweiternde Lücke zwischen der Herstellung von Tatsachen und der öffentlichen Verhandlung über die gesellschaftliche Wünschbarkeit der Herstellung der Tatsachen nicht nur eine Fortsetzung schon eingeschliffener Verfahrensund Verhaltensweisen, sondern ein spezifisches neues Grundlagenproblem sowohl für die Wissenschaften wie für die Gesellschaft." (S. 407).

\section{Anmerkung}

1) Zum kostenlosen Download unter http://hup.sub.uni-hamburg.de verfügbar.

$\ll 》$

\section{Denkanstöße und Perspek- tiven im Ringen um eine gerechtere Welt}

\section{Global Marshall Plan Initiative (Hg.): Hoffnung Europa. Strategie des Mitein- ander. Global Marshall Plan Foundation, Hamburg 2006, ISBN: 3-9809723-4-8, 320 Seiten, $12 €$}

\section{Rezension von Volker Stelzer, ITAS}

Von der ,Hoffnung Europa' zu sprechen, ist das nicht verwegen angesichts der verbreiteten Euroskepsis und eines angeblich drohenden „Kampfes der Kulturen'? Für die Herausgeber jedenfalls kann Europa als ein ,praktikables Beispiel für eine friedensfähige, sozial gerechte und nachhaltige Entwicklung für den gesamten Globus' genommen werden. Die 44 bekannten Persönlichkeiten aus Politik, Wissenschaft, Gesellschaft und Wirtschaft, die in dem Buch vertreten sind $^{1}$, wollen Denkanstöße zur weiteren Verbreitung der Vision geben, die Europa in ihren Augen für das zukünftige globale Miteinander der Staaten und Völker darstellen kann.

Alle Autoren des Buches teilen die Überzeugung, dass die derzeitig weitgehend konsistent Orientierung der staatlichen Systeme, der Wirtschaft und der Gesellschaft am neoliberalen Gesellschaftsmodell nicht zu einer Lösung der bestehenden globalen Probleme beiträgt (wie Armut, Ungerechtigkeit und Umweltzerstörung). Dies trauen die Herausgeber dem von ihnen entwickelten Modell eines „Global Marshall Plan“ zu, der am Ende des Buches vorgestellt wird: Nach ihrer Überzeugung bietet er durch den Aufbau einer ökosozialen Marktwirtschaft die Grundlage für eine faire Gestaltung der Globalisierung.

Das Buch begründet in den Kapiteln „Europäische Werte“, „Kultur des Miteinander“, „Bürgernähe und Partizipation“ und „Zukunft der EU“ den Modellcharakter Europas, den es in den Augen der meisten Autoren hat, um dann in den folgenden zwei Kapiteln mit der „Ökosozialen Marktwirtschaft“ und der „Globalisierung Made in Europa" Entwürfe für eine zukünftige Rolle Europas in der Welt vorzustellen. Die einzelnen Kapitel werden durch Kurzstatements bekannter Persönlichkeiten wie Bundespräsident Horst Köhler, Bundeskanzlerin Angela Merkel oder Bischöfin Margot Käßmann eingeleitet. Die Rezension stellt aus jedem Themenbereich mindestens einen Beitrag näher vor.

\section{1 „Glaubwürdigkeit" - ein wichtiger Europäischer Wert}

Interessant ist hier der Blick eines „NichtEuropäers" auf Europa: derjenige des Prinzen El Hassan bin Talal, dem Präsidenten des Club of Rome. Er hebt hervor, dass es Europa nach dem Schrecken des Zweiten Weltkrieges geschafft habe, sich zu einem Kontinent zu entwickeln, in dem die Länder eine gleichberechtigte Partnerschaft aufgebaut haben, in dem aus Feinden Verbündete wurden und Solidarität die Teilung abgelöst hat. Außerdem verweist er auf die Summe von 1 Mrd. Euro, die Europa monatlich für Hilfsprogramme auf allen fünf Kontinenten ausgibt. Er konstatiert, dass sich Europa einer hohen Glaubwürdigkeit erfreut und fordert es auf, diese Glaubwürdigkeit für Partnerschaften mit anderen Staaten einzusetzen. Diese Partnerschaften sollten sich vor allem auf die Themen 